\title{
Des systèmes cyber-physiques logistiques pour le déploiement de l'Internet Physique : application du paradigme holonique au transport auto-organisé et intercontinental de conteneurs
}

\section{Cyber-physical logistics systems for the Physical Internet deployment: application of the holonic paradigm to self-organized and} intercontinental containers transport

\author{
Fouzia Ounnar ${ }^{1}$, Patrick Pujo ${ }^{1}$ \\ ${ }^{1}$ Aix Marseille Université, CRET-LOG, Aix-en-Provence, France, fouzia.ounnar@univ-amu.fr, patrick.pujo@univ-amu.fr
}

RÉSUMÉ. L'Internet Physique va induire des changements organisationnels tandis que les Cyber-Physical Systems vont conduire à des changements technologiques majeurs. Leur conjonction nous permet de proposer des Cyber-Physical Logistics Systems sur la base d'une approche holonique d'auto-organisation des acteurs. Après une description des concepts et paradigmes à la base de cette proposition, cette dernière est détaillée. Un modèle structurel et décisionnel est ainsi décrit. La validation sera effectuée via un démonstrateur qui est présenté.

ABSTRACT. The Physical Internet will induce organizational changes while Cyber-Physical Systems will lead to major technological changes. Their combination allows us to propose Cyber-Physical Logistics Systems based on a holonic approach of self-organization of actors. After a description of the concepts and paradigms underlying this proposal, the latter is detailed. A structural and decisional model is thus described. Validation will be carried out via a demonstrator which is presented.

MOTS-CLÉS. paradigme holonique, internet physique, systèmes cyber-physiques logistiques, aide multicritère à la décision.

KEYWORDS. holonic paradigm, physical internet, cyber-physical logistics systems, multicriteria decision support.

\section{Introduction}

La révolution industrielle actuelle, appelée 'Industry 4.0' [KAG 13], se base sur une interconnexion forte des objets et/ou des acteurs mais également du monde réel et du monde virtuel via les innovations des TIC (technologies de l'information et de la communication) [ELK 16]. Ceci touche plus largement l'écosystème industriel et va impacter le monde de la logistique, qualifiée déjà de 'Logistique 4.0'. En effet, selon $[\mathrm{KOH} 15]$, la révolution numérique efface progressivement les limites entre le BtoB (Business to Business) et le BtoC (Business to Customer), en interconnectant directement offres et demandes. Le collaboratif interactif et transparent offert par les évolutions du monde de l'Internet fait disparaitre l'organisation traditionnelle en filières, branches et métiers, et fait pénétrer le client au sein de l'élaboration de la valeur ajoutée, au sein d'une relation de co-conception hybride clientfournisseur. Dans ce contexte, l'avantage ira à celui qui saura, en associant ses clients et en leur offrant de nouvelles possibilités, faire naître des possibilités insoupçonnées issues de business complémentaires.

Les sauts technologiques constatés ces dernières années, liés notamment au développement des outils numériques de communication, ont conduit à des changements de paradigmes qui doivent intégrer ces nouvelles donnes. Dans le monde du manufacturing, l'industrie 4.0 repose sur diverses avancées technologiques, parmi lesquelles nous pouvons citer principalement les CPS (cyber physical systems) [LEE 08], l'IoT (internet des objets) [SAL 18], et l'IoS (internet des services) [HER 16], 
[KAG 13]. L'IoT représente une infrastructure intelligente des TIC qui permet la communication et la coopération en temps réel entre les objets (machines et dispositifs) ainsi que la connexion entre le monde physique et virtuel [TAO 17] dans un environnement dynamique connecté, donnant accès à des services distants (IoS). Un CPS est un système embarqué autonome, doté de capteurs pour percevoir son environnement, capable d'agir sur des processus physiques au moyen d'actionneurs. Les CPS sont connectés les uns aux autres via des réseaux digitaux et ils sont aptes à utiliser des services distants pour les assister. Ces différents concepts peuvent globalement être considérés comme des extension de la robotique et de l'automatisation, allant dans la sens de la digitalisation du monde [NOY 16].

Les CPS permettent donc une interaction instantanée et continue entre des éléments physiques et des éléments virtuels et également avec des acteurs extérieurs. Les objets deviennent de plus en plus autonomes et les systèmes de plus en plus reconfigurables du fait que l'accès à l'information devient possible à tout moment et de partout. S'ajoute également à cela la capacité d'intelligence donnée aux objets et aux systèmes, ou la capacité d'aller solliciter via l'aptitude de communication des services distants aptes à fournir cette intelligence. Dans ce contexte, l'Industrie 4.0 conçoit l'usine, voire la filière de production dans son ensemble, comme un gigantesque système cyber-physique largement autorégulé par des interactions machine-objet. Il en est attendu des gains en termes de flexibilité et d'agilité, des gains de temps, de qualité et de coûts [KOH 15]. Il en découle le concept de cyberphysical production systems (CPPS) [MON 14]. En corollaire avec la transformation en cours du monde du manufacturing, l'organisation du secteur de la logistique doit aussi évoluer.

L'objectif est de permettre une meilleure mutualisation des ressources logistiques, par l'interconnexion de plusieurs acteurs logistiques, pour une meilleure performance, en termes d'efficacité, d'efficience et de qualité de service. Ceci aboutit au concept de cyber-physical logistics system (CPLS). Les CPLS sont à la convergence de différents thèmes de recherche scientifique, comme l'opérationnalisation du paradigme holonique [PUJ 16] et/ou celle du cadre fourni par le concept d'Internet Physique [BAL 14]. Ce concept permet de sortir la logistique de son mode actuel de croissance tout azimut, en lui offrant un cadre vertueux de développement, et offre un scénario soutenable à l'horizon des prochaines décennies [HOU 15].

Cela nous conduit à la question de recherche suivante : l'auto-organisation du pilotage de systèmes logistiques via des microsystèmes embarqués est-elle une solution possible pour le fonctionnement rationnel de l'Internet Physique?

Dans cet article, nous envisageons l'usage de CPLS pour opérationnaliser l'Internet Physique, en nous appuyant sur le paradigme holonique. Pour cela, le prochain paragraphe proposera un panorama des concepts et paradigmes en relation avec ce travail de recherche. Ensuite, nous détaillerons notre proposition, tant sur le plan de la modélisation structurelle et architecturale que sur celui de la modélisation décisionnelle, d'un pilotage d'un système logistique global fondé sur l'aide multicritère à la décision, auto-organisée et dynamique. Puis, nous poserons les tenants du démonstrateur sur lequel nous travaillons actuellement pour valider ces propositions. Enfin, une conclusion clôturera cet article et présentera les perspectives.

\section{Positionnement scientifique de la proposition}

Nous allons ici rappeler les concepts et paradigmes sur lesquels s'appuie notre proposition.

\subsection{Internet Physique : une vision pour la logistique du futur}

La demande logistique s'est considérablement développée ces dernières décennies [FUL 11]. Si la performance des acteurs de la logistique est effectivement au rendez-vous de ce besoin croissant, cela n'est pas sans impacts négatifs sur l'environnement et la société : l'efficience n'est pas bonne. En effet, les organisations logistiques actuelles expédient beaucoup d'air et d'emballage. 
L'Internet Physique vise à mettre en place un système logistique global ouvert, efficient et durable. [MON 11] propose une remise en question des paradigmes habituellement utilisés en logistique, tant aux plans locaux, régionaux que planétaire. Ce système logistique, qui englobe la réalisation, le déploiement et la livraison de biens physiques, se met en place au travers de réseaux interconnectés et exploite un ensemble standard de protocoles collaboratifs de routage, de conteneurs modulaires et d'interfaces intelligentes. L'effet d'échelle et la mutualisation des ressources de transport permettent d'atteindre une meilleure efficience du système global. Tout ceci doit pouvoir se faire dans des conditions économiques équitables pour les différents acteurs intervenant dans le processus d'acheminement de chaque marchandise.

Ce concept fait l'objet d'un intérêt scientifique majeur dans de nombreux pays, et divers projets de recherche sont engagés. Si l'Internet Physique s'inspire de la gestion des flux de données pour mettre en œuvre les flux logistiques, ce qui prône dans une certaine mesure le pilotage par les flux, il doit avant tout viser à l'optimisation de ces flux (le transport de biens matériels est bien plus onéreux que le transport d'informations). Partant de ce constat, il semble naturel de regarder les travaux liés à la modélisation des flux de produits et des traitements d'informations de prise de décision en manufacturing, afin de trouver les formalismes les plus adéquats à la représentation de tels systèmes complexes, au sens de [LEM 90] et de [OLI 97]. Pour cela, nous nous sommes intéressés au paradigme holonique.

\subsection{Paradigme Holonique : une approche pour la modélisation de la complexité logistique}

Pour revisiter la notion de décomposition hiérarchique des systèmes (Open Hierarchical Systems Theory), le philosophe hongrois Arthur Koestler a proposé l'assertion suivante : tout système doit être observé comme une hiérarchie multi-niveaux de sous-ensembles semi-autonomes formant des arborescences verticales et des réseaux horizontaux [KOE 67]. L'élément de base de cette approche est appelé 'holon'. Un 'holon' est une partie identifiable d'un système ayant une identité unique, mais qui pourtant se compose de pièces subalternes et qui est également une partie d'un plus grand ensemble [OLI 97]. Ces deux aspects du holon sont fondamentaux, car il montre la dualité fondamentale, au niveau fonctionnel et au niveau comportemental, d'un holon vis-à-vis d'un simple système. Le premier de ces aspects confère au holon l'identité propre d'un tout autonome, c'est-à-dire existant par lui-même (Self-Assertative tendencies). Par ailleurs, le holon se trouve être une partie intégrée dans un tout (Integrative tendencies). Sur ce second aspect, l'intelligence associée au holon doit lui permettre de contribuer pour une partie au fonctionnement global du système.

Différentes architectures holoniques ont été proposées dans le cadre de l'étude des Holonic Manufacturing Systems (HMS) [DEE 03]. Si la plus connue reste PROSA (Product, Resource, Order, Staff Architecture) [VAN 98], d'autres architectures sont relativement semblables, comme Adacor (ADAptive holonic COntrol aRchitecture for distributed manufacturing systems) [LEI 06], MetaMorph [BAL 01] ou PROSIS (Product, Resource, Order, Simulation Isoarchic Systems) [PUJ 09]. Dans toutes ces architectures, le holon se compose d'une partie 'traitement de l'information' (le I_holon) et d'une partie 'structure physique' (le M_holon) [PUJ 12] (Cf. Figure 1). Le I_holon dispose d'un système d'information contenant des données relatives au $\mathrm{M}_{-}$holon et d'une intelligence décisionnelle qui aide à contrôler le M_holon. 


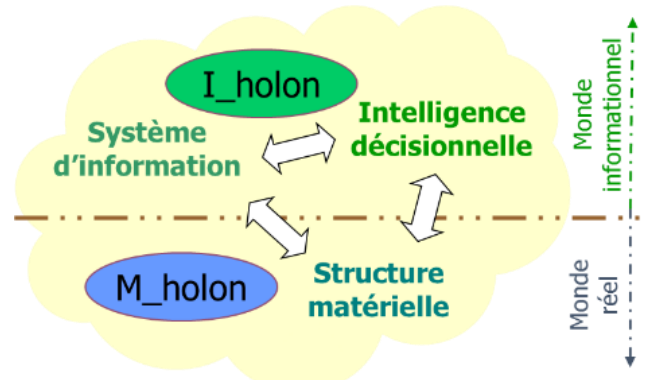

Figure 1. Structure interne d'un holon : association d'un I_holon et d'un M_holon

Ainsi, le holon peut agir sur son propre comportement, mais il peut également d'intervenir sur le comportement du système auquel il appartient. En d'autres termes, un holon a l'autonomie suffisante pour créer et contrôler l'exécution de ses propres plans, et il peut coopérer avec d'autres holons pour développer des plans mutuellement acceptables, afin de réaliser un objectif global à tout le système de production. Le concept de holon permet donc d'associer de l'intelligence à l'entité qu'il modélise. Ces propriétés permettent de dessiner les contours d'une modélisation holonique des CPLS.

\subsection{Cyber-Physical Logistics Systems : une opportunité technologique pour la mise en œuvre}

Les CPLS (Cyber Physical Logistics System) sont l'émanation des CPPS, appliqués à la logistique. Les CPPS sont eux-mêmes des systèmes cyber physiques (CPS) mis en œuvre dans le cadre d'une production manufacturière [MON 14]. Selon [KOH 15], les CPS regroupent des systèmes embarqués qui utilisent des capteurs pour récupérer des données et qui agissent sur des processus physiques au moyen d'actionneurs. Ils sont connectés les uns aux autres via des réseaux digitaux, utilisent toutes données et services disponibles mondialement et bénéficient d'interfaces hommes-machines multimodales.

L'explosion de l'intérêt pour les CPS est tout d'abord corrélé avec la capacité d'embarquer une capacité de traitement temps réel qui permet soit une forte interaction avec l'usager du CPS, soit une totale prise en compte en temps réel de l'environnement permettant une automatisation intelligente du CPS. Ceci permet de créer de nouveaux produits, capables d'embarquer des fonctionnalités leur conférant une attractivité marketing justifiant le surcoût par rapport à des produits plus conventionnels.

De fait, l'émergence de nouveaux concepts ICT a profondément transformé la recherche sur les systèmes industriels [TRE 16], ouvrant la porte à des propositions qui semblaient auparavant impossibles. Ainsi, parmi différents challenges majeurs, [ELK 16] a identifié que cela permettait d'offrir des capacités contextuelles nouvelles dans des environnements d'entreprise complexes. Nous sommes effectivement totalement convaincus que le fait de distribuer de l'intelligence et de la capacité de décision apporte énormément d'avantages dans le cadre de CPS dédiés à des systèmes de production ou à des systèmes logistiques. Ce type d'approches a été longuement étudié par anticipation par de nombreuses équipes de recherche : [CAR 17] dresse un panorama des différents travaux conduits en France autour des Intelligent Manufacturing Systems. [MON 06], [LEI 09] et [PUJ 12] proposent chacun un large panorama des solutions de pilotage distribuées, fondées sur les systèmes multi agents et/ou le paradigme holonique. Par exemple, [PUJ 16] propose de construire des CPS pour le pilotage d'atelier et le pilotage de système de transitique en utilisant des composants de réseaux de capteurs sans fil, constituant ainsi une instance de CPPS [MON 14] [MON 15]. [LEI 16] propose le concept très proche de CPS industriels et a identifié 27 challenges clefs pour leur déploiement, évaluant pour chacun leur difficulté, leur priorité et leur horizon de maturité. Ceci peut être extrapolé aux CPLS. Le concept de CPLS a été initialement introduit dans une revue chinoise d'audience nationale en 2011 par [LAI 11]. Un CPLS est un type spécial de CPS qui effectue des tâches logistiques [THO 14], avec des objectifs économiques, écologiques et sociaux. 
En cela, les CPLS apparaissent comme une réponse technologique possible pour la mise en œuvre de l'Internet Physique. Ainsi, [OUN 16] ont posé les bases d'un CPLS en proposant une approche générique de détermination dynamique de la chaîne logistique de transport de produits, depuis le premier kilomètre jusqu'au dernier kilomètre. Cette approche du pilotage, développée ici, utilise des entités logistiques intelligentes qui s'appuient sur une prise de décision multicritère pour apporter des réponses pertinentes à la complexité des choix de fonctionnement possible. Cela vise à la mise en place d'un transport de marchandises entièrement automatisé [VER 01].

\subsection{Aide Multicritère à la Décision : une solution pour absorber la complexité}

Parmi les nombreuses méthodes d'aide multicritère à la décision [DOU 02], [ISH 13], nous avons choisi la méthode ANP (Analytic Network Process) [SAA 96] qui présente de très nombreux avantages. ANP décompose un problème complexe de décision en un ou plusieurs niveaux de détails où l'évaluation des valeurs est fournie par des comparaisons par paires, en prenant en considération un mix de critères qualitatifs et quantitatifs. Une structure hiérarchique formalisant la prise de décision (type arbre) peut être enrichie par des liens transversaux (type réseau) (Cf. Figure 2), l'ensemble des liens étant pondérés et différents jeux de pondération pouvant être inter changés.

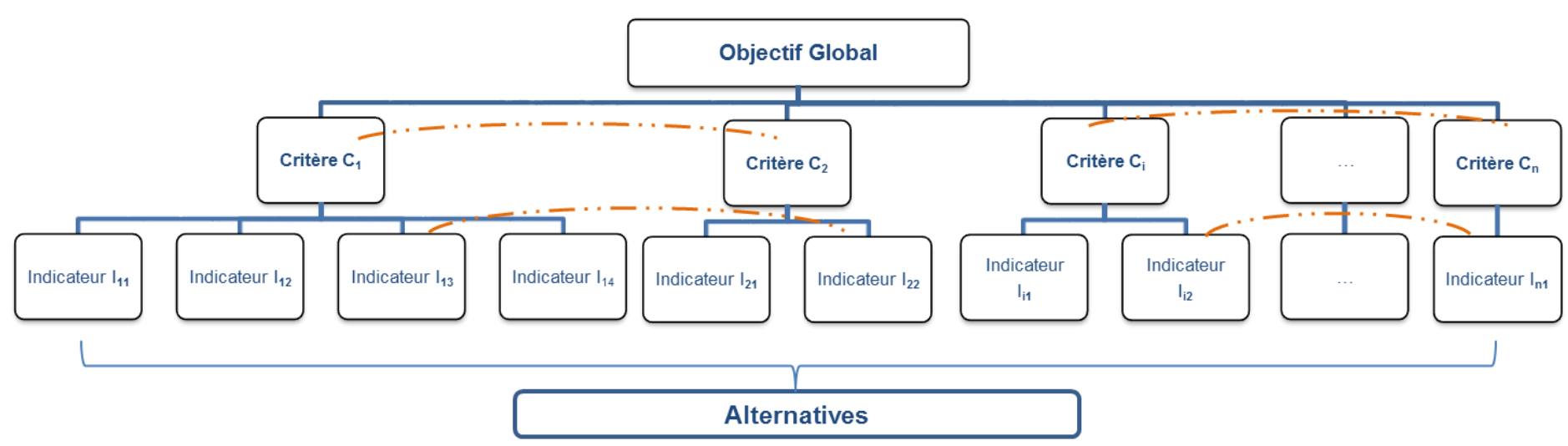

Figure 2. Structure décisionnelle ANP

Par ailleurs, ANP permet de valider la cohérence des préférences du décideur dès la conception du réseau décisionnel (c'est-à-dire son paramétrage), puis son exécution automatique, en temps réel, lors de l'exploitation (c'est-à-dire lors du pilotage).

Cet ensemble de connaissances permet de proposer un pilotage auto-organisé de l'Internet Physique.

\section{Proposition d'un Pilotage auto-organisé de l'Internet Physique}

Après un rapide survol du contexte nous ayant amené à faire cette proposition, nous en proposons un modèle formel ainsi que quelques solutions visant à une mise en œuvre pratique.

\subsection{Cadre de la proposition : le pilotage auto-organisé}

Les solutions de pilotage centralisées sont généralement fondées sur la recherche d'un optimum à partir du regroupement de données collectées antérieurement sur le terrain ou définies de manière prévisionnelle. Nos travaux de recherche sur le pilotage de systèmes manufacturiers complexes nous ont permis de constater que ces solutions n'étaient pas robustes face aux perturbations inhérentes au fonctionnement propre du système opératoire et aux variations des sollicitations provenant de la dynamique de l'environnement. La solution optimale n'est généralement pas applicable, car elle ne correspond pas aux conditions réelles de fonctionnement. Nous défendons l'idée qu'une solution basée sur un compromis utilisant des données obtenues en temps réel, et donc sures, est plus intéressante que 
des solutions recherchant des optimums utilisant des données prévisionnelles appelées à devenir potentiellement obsolètes.

Par ailleurs, l'approche auto organisée que nous proposons est basée sur un accroissement de l'autonomie des 'acteurs' (acteur : entité identifiable ayant un rôle ou un intérêt dans le fonctionnement du système opératoire). Il s'agit d'accroitre leur capacité à décider de manière intelligible, et leur capacité à communiquer et à collaborer, afin que chaque acteur puisse prendre en compte dans la prise de ses décisions l'état de son environnement. L'objectif est de développer des qualités de réactivité, ce qui est rendu possible si les décisions sont prises en fonction de l'état réel de chaque acteur. Pour ce faire, chaque acteur doit être doté d'un centre de décision Temps Réel lui permettant de participer à une négociation par appel d'offres sur la base d'une analyse multicritère de sa propre performance. Chaque acteur exploite le même mécanisme de décision multicritère, cependant chaque acteur est décrit par un paramétrage et des variables d'état qui le décrivent en propre, et qui lui permettent de se différentier vis-à-vis d'un ensemble de critères : ceci lui permet de participer à la négociation en mettant en évidence les appels d'offres pour lesquels il est le plus performant et/ou qui lui sont potentiellement le plus profitable. La prise de décision multicritère permet d'intégrer des points de vue différents, voire antagonistes, tous contribuant à la construction de la solution de pilotage. Cette solution de pilotage émerge donc au niveau global de l'agrégation d'une succession de comparaisons multicritères effectuées au niveau local.

Nous appliquons ici ces principes au secteur de la logistique et de l'Internet Physique, dans la lignée de travaux antérieurs de [PUJ 09], [PUJ 16]. Le pilotage isoarchique des relations liant chacun des partenaires permet de mettre en place une auto-organisation des fournisseurs/sous-traitants/prestataires logistiques/transporteurs pour répondre au mieux aux besoins des clients. Visant à augmenter le fonctionnement dynamique de la supply chain dans un cadre formel et collaboratif et compatible avec des réseaux de prestataires logistiques ou des réseaux de réseaux, cette proposition constitue une vision innovante de l'Internet Physique, vis-à-vis des solutions plus classiques proposées dans [BAL 14]. Au fur et à mesure (temps réel à l'échelle d'un système logistique) de l'apparition de chaque nouveau besoin de fourniture (achat ou sous-traitance) exprimé par l'un des clients, un fournisseur/soustraitant/prestataire logistique/transporteur va être recherché pour répondre à ce besoin : pour cela, un appel d'offre est émis dans le réseau. Les réponses, élaborées par chaque acteur du réseau potentiellement en état de répondre via une analyse multicritère, sont gérées via un mécanisme d'enchères. La meilleure réponse indique l'affectation de la tâche correspondante à l'acteur l'ayant exprimé. Cette approche auto-organisée du fonctionnement des acteurs du réseau logistique global qu'est l'Internet Physique favorise la réactivité, le lissage des charges et une haute qualité de service.

L'auto-organisation sous-entend que les décisions de pilotage doivent être prises localement. L'idée générale est que seules les entités concernées par une décision puissent contribuer à l'élaboration de cette décision, et qu'elles le fassent avec un maximum d'expertise et de connaissances sur leur propre état. Cette prise de décision permet d'assurer une sélection de l'acteur qui sera le plus fiable et qui répondra au mieux aux besoins du client [OUN 08].

Dans le cadre de la logistique, les réseaux logistiques fonctionnent actuellement autour de chaines logistiques assez figées, ce qui génèrent beaucoup de voyages à vide, des installations d'entreposage mal utilisées, des produits non stockés aux bons endroits, des déplacent inutiles de produits, des transports inefficients et insoutenables. Il est à noter que l'élaboration de solutions localement optimales est effectuée dans le respect d'un certain nombre de critères globaux de coopération. Par exemple, une vérification et éventuellement un ajustement de la capacité collective du réseau face à la charge prévisionnelle sont à considérer périodiquement.

Cette orientation autour de l'auto-organisation est complétée par l'utilisation de l'aide à la décision multicritère. Cette association constitue une part originale de l'approche, et contribue sûrement à sa performance. Le choix de la méthode décisionnelle multicritère localement mise en œuvre, ANP en 
l'occurrence, a été effectué sur son aptitude à être automatisée, c'est-à-dire sur la possibilité de mettre en œuvre la méthode en temps réel, sans intervention et interaction humaine, lors du pilotage du système logistique.

Par ailleurs, ce choix a été validé par le biais d'une étude comparative (aide multicritère à la décision, locale temps réel, vs. optimisation, globale, centralisée), qui a confirmé les performances obtenues [OUN 15]. Cette étude s'est appuyée sur des benchmarks académiques reconnus dans le domaine. L'analyse des résultats montre que la proposition permet d'obtenir des résultats équivalents voire meilleurs.

Enfin, les décisions locales sont prises à un niveau proche des perturbations. Ceci permet d'obtenir plus de réactivité et d'agilité. Si un problème apparait, les acteurs concernés rechercheront la meilleure solution en fonction de leurs états réels. En fait, l'approche proposée consiste en un parti pris visant à privilégier l'enchainement de bonnes décisions locales opportunistes par rapport à l'obtention d'une solution globale théoriquement optimale, mais peu robuste face à des perturbations qui ne manqueront pas d'apparaitre et de venir contrarier le déroulement de cette solution optimale [PUJ 12]. Du point de vue du pilotage du système logistique, la perturbation devient vécue comme un événement normal.

\subsection{Formalisation structurelle : une modélisation holonique des CPLS}

Nous pouvons illustrer le principe ainsi énoncé dans le cas d'un transport de marchandises. Prenons le cas d'un produit ' $p_{x}$ ' devant aller du point 'A' (Entreprise A) au point 'B' (Entreprise B) (Cf. Figure 3). Ce produit va tout d'abord être emballé (stock de sortie de A 'SS_A') dans un carton 'Ca_1', qui va lui-même être envoyé dans un conditionnement ' $\mathrm{Ca} 2$ ' ' par la voie routière 'Transport_1'vers un point ' $\mathrm{C}$ '. 'Ca_2' sera à son tour rangé ou conditionné à l'intérieur d'un conteneur 'Co_1' qui va être envoyé par voie maritime 'Transport_2' jusqu'à un point ' $D$ '. A ce niveau, les différents conditionnements sont triés ou reconditionnés pour être envoyés aux clients. Le carton ' $\mathrm{C} 1$ ' comportant le produit ' $\mathrm{p}_{\mathrm{x}}$ ' va donc être acheminé vers le point ' $\mathrm{B}$ ' par le biais d'un camion 'Transport_3' dans un conditionnement 'Ca_3' (qui arrivera en stock d'entrée de B ('SE_B').

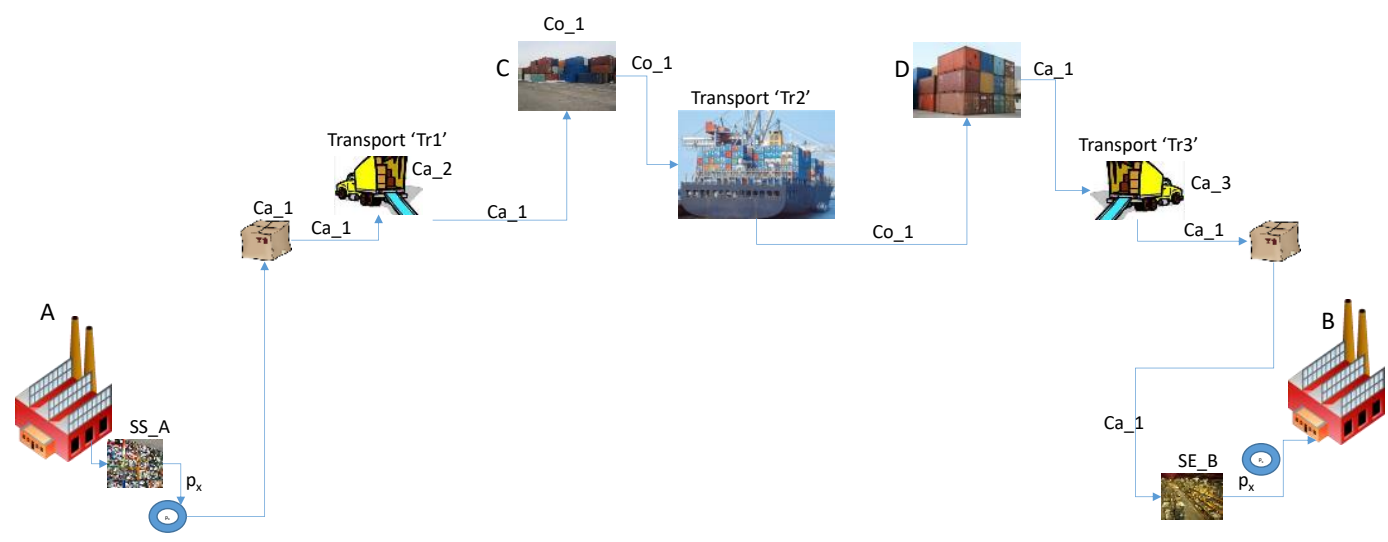

Figure 3. Cheminement du produit ' $p_{x}$ ' de 'A' vers ' $B$ '

Dans le but de mettre en œuvre le transport du produit ' $p_{\mathrm{x}}$ ' du point A au point B et de pouvoir suivre son déroulement, il est nécessaire de mettre en place, tout au long de chaque chaîne logistique faisant parti du réseau logistique, un système de groupage et de dégroupage de différents ordres de transports, concernant différents produits et nécessitant différentes ressources.

A partir du réseau de transport distribué et ouvert d'informations qu'est Internet, la transposition du concept au transport des objets et donc aux activités logistiques permet de proposer une véritable révolution logistique, articulée autour d'un système logistique mondial, ouvert et exploitant des réseaux de transport et d'approvisionnement interconnectés via des hubs intermodaux, utilisant des 
protocoles collaboratifs et des conteneurs standardisés modulaires et adressable via des interfaces intelligentes standards.

Concernant cette approche auto-organisée d'Internet Physique, les problématiques de choix logistique correspondent finalement à gérer, au niveau de chaque nœud (PI-hub) de la chaîne logistique faisant partie d'un réseau logistique, d'une part la convergence de flux en entrée de ce nœud et d'autre part la divergence de flux en sortie de ce nœud (Cf. Figure 4).

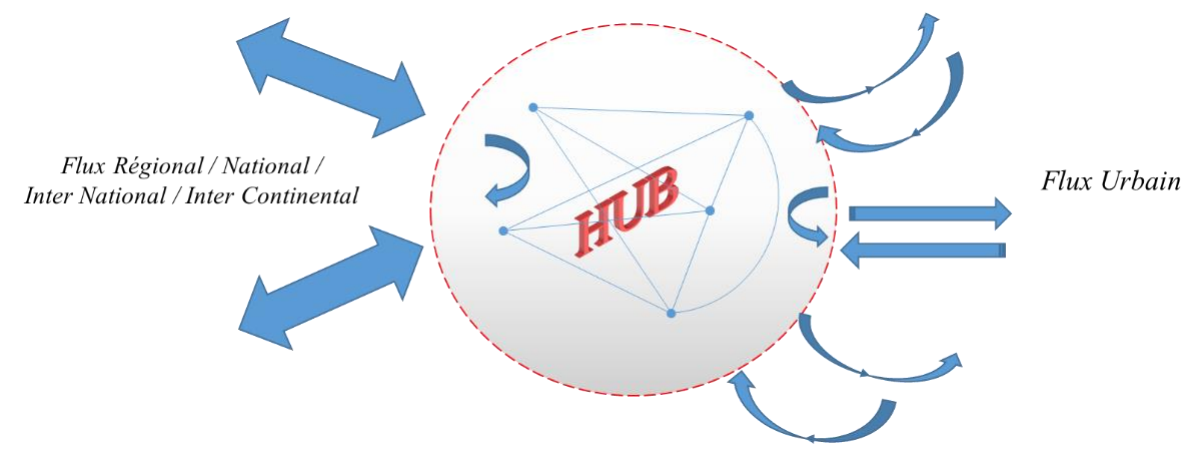

Figure 4. Plateforme de mutualisation : le rôle fondamental des hubs

Chaque hub réalise ainsi un changement d'échelle dans le mode de transport utilisé : du détail vers du volume en début de transport, du volume vers du détail quand on se rapproche de la livraison (Cf. Figure 5). Quel que soit le niveau de granularité du hub dans le réseau logistique (hub urbain, régional, national, international ou intercontinental), il s'agira de gérer des produits, des ressources et des ordres. En effet, les flux entrants/sortants d'un nœud peuvent être de nature différents (petits et/ou grands flux (volumes)), nécessitant des ressources de types différents (camionnettes, camions, trains, bateaux, avions...) et devant répondre à des missions spécifiques (transport d'un produit d'un point $\mathrm{A}$ à un point $\mathrm{B}$, avec ou sans date précise de livraison...), mais le rôle du hub reste fondamentalement le même.

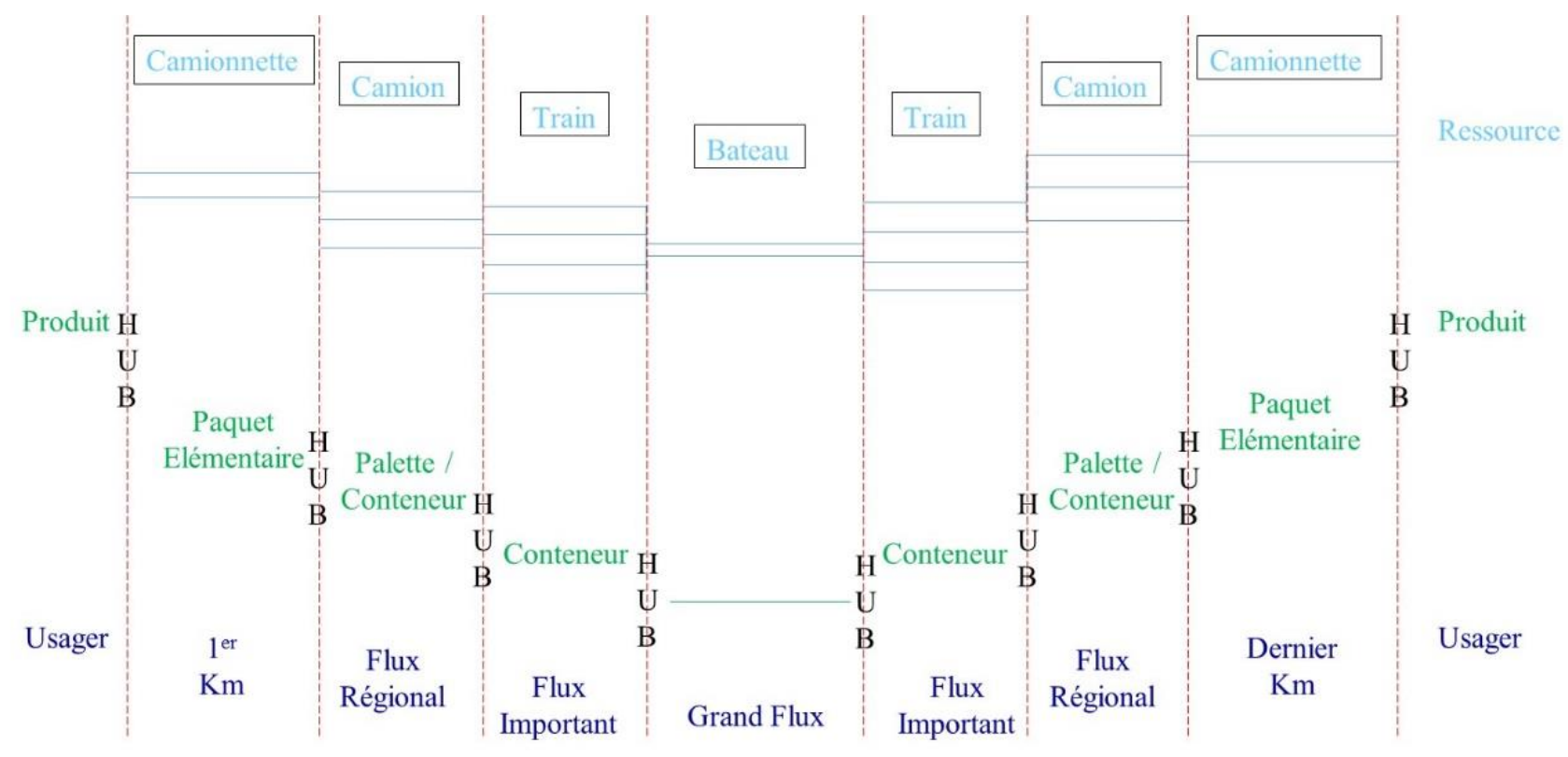

Figure 5. Plateforme de mutualisation : le rôle fondamental des hubs

L'objectif est de pouvoir traiter ainsi la totalité de la chaîne (B2B / B2C / C2C) avec des méthodes décisionnelles et des critères fonctionnels homogènes. Notre proposition consiste à déterminer la chaîne logistique de transport de produits, de manière générique et en temps réel, depuis le premier kilomètre jusqu'au dernier kilomètre, en exploitant certains aspects propres à l'Internet Physique, tels que l'encapsulation des marchandises. La même approche, générique, permet de traiter des 
problématiques de choix pour le transport de masse transcontinental jusqu'à celles de la mutualisation du dernier kilomètre en logistique urbaine.

L'exploitation de cette approche de l'Internet Physique aura donc des implications importantes sur l'évolution des différents types de logistique (urbaine, régionale ...) en termes d'homogénéisation et de standardisation.

Comme dans les HMS [PUJ 12] et à tous les niveaux de la chaine logistique, nous pouvons observer que le plus petit dénominateur commun entre les holons impliqués, souvent extrêmement différents, réside dans un noyau de trois types d'entités holoniques :

- un type de holon associé à chaque marchandise étant traité par le système logistique en question ;

- un type de holon associé à chaque équipement/ressource entrant dans ce traitement ;

- un type de holon associé au traitement en question, et donnant les caractéristiques de la mission ou de la tâche associée.

Il s'agit des trois types classiques de holons \{Produit, Ressource, Ordre\} [VAN 98], [PUJ 12].

La problématique est alors la suivante : pour atteindre une destination, il existe plusieurs chemins possibles. Chaque chemin est constitué d'une succession de segments. L'ensemble des segments constitue le réseau de transport. Il existe potentiellement plusieurs ressources pour effectuer un transport sur un segment donné et dans une plage horaire donnée. La connexion entre les segments successifs se fait dans un hub, avec à la clef un éventuel changement de ressource de transport [OUN 16]. Il faut donc choisir l'ensemble des ressources sur les différents segments d'un chemin pour assurer la date de livraison d'un produit, ou un retard minimal.

Si ce problème semble correspondre à un problème classique d'optimalisation, ce type de solutions théoriques n'est ici pas vraiment adapté, principalement pour différentes raisons. Premièrement, à l'échelle d'un système logistique mondial du type de l'Internet Physique, la taille du problème est très importante et les temps d'obtention d'une solution sont donc très longs. Par ailleurs, la modélisation du problème change à l'apparition de chaque nouveau transport. Il faudrait donc figer le jeu de données sur des fenêtres temporelles (ne plus accepter de nouvelles missions de transport durant un certain temps) pour obtenir une solution efficace. Enfin, les missions de transport sont matériellement longues : elles dépendent de la vitesse des ressources de transport utilisées. Il y a donc tout le temps pour que des aléas et des perturbations apparaissent, et que la solution prévisionnelle initialement optimale s'avère en fait beaucoup moins performante. Tout ceci interdit une grande réactivité et va à l'encontre des objectifs recherchés.

C'est pour cela que nous préférons étudier des solutions d'auto organisation, basées sur des collaborations opportunistes visant à obtenir un bon compromis entre les objectifs propres entre les différentes entités holoniques en interaction.

Le Holon Produit (HP) modélise l'objet à transporter, c'est-à-dire la marchandise. Comme dans PROSIS [PUJ 09], il existe un $\mathrm{HP}_{\mathrm{i}}$ par marchandise à transporter, peu importe sa nature. Il peut être tout à fait possible qu'un HP représente un lot de produits, si l'ensemble des produits de ce lot a la même destination et est associé dans un même conditionnement. Ceci est évidemment important pour les produits de petite taille et de faible valeur. Hormis ses caractéristiques intrinsèques, un HP sera caractérisé par ses dimensions, ce qui permettra de lui trouver le container ad hoc pour son transport, par sa destination finale et par sa date de livraison souhaitée. Ce holon a un rôle en apparence passif, mais il contribue en fait à l'évaluation de performance, par exemple en terme de qualité de service individuelle, et il sert de lanceur d'alerte en cas de contre-performance (date de livraison risquant d'être dépassée, ou pire, retard avéré). 
Le Holon Resource (HR) modélise le moyen de transport. A chaque moyen (camion, bateau, train...) correspond un $\mathrm{HR}$. Chaque $\mathrm{HR}_{\mathrm{j}}$ a comme objectif de remplir au maximum sa capacité : nous sommes bien dans le cadre de l'objectif de l'Internet Physique, qui vise à réduire les circulations à vide. Il va donc de soi que ceci ne peut être obtenu que dans le cadre d'une mutualisation globale des ressources, en séparant clairement les notions d'opérateurs et de transitaires, et donc en répartissant équitablement les bénéfices entre les différents acteurs au prorata de leurs coûts et de la valeur ajoutée induits. Ce principe permet d'utiliser sans problème les ressources présentes, avec comme seule préoccupation leur rentabilité opérationnelle.

Le Holon Ordre (HO) est celui qui finalement gère le transport effectivement réalisé. En fait, chaque $\mathrm{HO}_{\mathrm{k}}$ est porté par un PI-container [SAL 15] qui est un conteneur physique utilisé pour encapsuler des objets physiques. Il hérite des caractéristiques du $\mathrm{HP}_{\mathrm{i}}$ associé : lieu et date de livraison. Son objectif est de s'associer à d'autres PI-containers pour constituer un container correspondant aux tailles standards de manutention dans les Hubs. En effet, au cœur du fonctionnement de l'Internet Physique, l'agrégation / désagrégation de PI-containers sur des segments de transport permet de réaliser des économies d'échelle et d'atteindre une meilleure rentabilité de chaque utilisation de ressource. C'est d'ailleurs là que réside tout l'intérêt de l'Internet Physique.

Lors de l'association de 2 PI-containers (ou plus), lors de relations d'encapsulation ou de composition, l'ensemble des $\left\{\mathrm{HO}_{\mathrm{k}}\right\}_{\mathrm{m}}$ concernés est alors représenté par un $\mathrm{HO}_{\mathrm{m}} \cdot \mathrm{Ce} \mathrm{HO}_{\mathrm{m}}$ peut être l'un des $\left\{\mathrm{HO}_{\mathrm{k}}\right\}_{\mathrm{m}}$ qui prend le rôle de représentant des autres $\mathrm{HO}$. A l'arrivée dans un $\mathrm{Hub}, \mathrm{le}_{\mathrm{HO}}$ vérifie si les $\mathrm{HO}_{\mathrm{k}}$ qu'il représente continuent à aller dans la même direction, c'est-à-dire s'ils vont transiter sur le même segment $\mathrm{S}$ de sortie. Si tel est le cas, le $\mathrm{HO}_{\mathrm{m}}$ recherche une ressource de transport pour l'ensemble de ses $\left\{\mathrm{HO}_{\mathrm{k}}\right\}$. Sinon, il demande au Hub de lancer une procédure de désagrégation du $\mathrm{HO}_{\mathrm{m}}$ afin d'orienter chaque $\mathrm{HO}_{\mathrm{k}}$ vers le segment lui correspondant.

Une fois de nouveaux $\mathrm{HO}_{\mathrm{x}}$ reconstitués, chacun d'entre eux lance une procédure de recherche d'une ressource de transport ad hoc.

De nombreux travaux ont déjà été conduits sur ce type de problématique [PUJ 01], [BAL 12], [BAL 13], [OUN 15], [SAL 15]. Toutefois, c'est du comportement adopté par les holons dans les hubs que découlent la fluidité des flux et le lissage des charges, dont dépend l'obtention de la performance et de la durabilité du système logistique global.

\subsection{Formalisation fonctionnelle : interactions et aide à la décision isoarchique}

Avec l'approche de pilotage que nous proposons ici, les entités holoniques sont potentiellement toutes en interactions les unes avec les autres. En fait, seules les interactions utiles à la prise de décision vont réellement être mise en œuvre : les mécanismes de prise de décision se mettent en place au sein d'une holarchie, c'est-à-dire d'un sous ensemble de holons (d'un même type ou pas) qui doivent coopérer pour atteindre un objectif donné.

Par exemple, dans une mission de transport élémentaire, peuvent se retrouver dans une même holarchie :

- le holon produit, permettant de définir l'objet du travail (Que doit-on transporter ?) ;

- le holon ordre qui décrit la mission de transport de ce produit (Comment va être réalisé le transport? Quelle est la date de livraison au plus tard ?) ;

- les holons ressources correspondant aux moyens nécessaires pour réaliser la mission (Quels sont les moyens adéquats disponibles?).

Nous devons également considérer le fait qu'un holon est récursif. Un holon, appartenant à une holarchie, peut lui-même être une holarchie, qui se comportera comme une unité autonome et coopérative dans la première holarchie [PUJ 12]. 
Reprenons la modélisation du transport du produit ' $p_{\mathrm{x}}$ ' présenté à la figure 3 . Ce produit sera modélisé par un $\mathrm{HP}_{\mathrm{x}}$, composé de sa partie informationnelle (I_produit ${ }_{\mathrm{x}}$ ) et de sa partie physique (M_produit ${ }_{\mathrm{x}}$ : la marchandise à transporter). Un ordre de transport $\mathrm{HO}_{\mathrm{z}}$ sera associé au $\mathrm{HP}_{\mathrm{x}}$ : Ce sera le conteneur ${ }_{\mathrm{z}}$. Enfin, différents $\mathrm{HR}_{\mathrm{j}}$ seront affectés au transport du conteneur $\mathrm{HO}_{\mathrm{z}}$. Tout au long de cette chaîne logistique, chaque type de holon $\mathrm{HX}_{\mathrm{i}}$ sera encapsulé dans un $\mathrm{HX}_{\mathrm{i}+1}$, où ' $\mathrm{X}$ ' représente le produit ' $\mathrm{P}$ ' ou l'ordre ' $\mathrm{O}$ ' ou la ressource ' $\mathrm{R}$ '). Dans le cadre de cette modélisation récursive [PUJ 07], il sera nécessaire de gérer la complexité d'accès aux données.

Pour cela, l'architecture isoarchique permet, à chaque étape de prise de décision, de mettre en évidence uniquement les entités I_holons concernées par la décision : ce sont celles qui appartiennent à une même holarchie. L'émergence d'une solution de pilotage dans un système holonique est issue des interactions que ces holons ont su gérer. Dans notre proposition, l'ensemble des HR et des HO se met au service des HP, qui fournissent chacun une part du cahier des charges des transports à réaliser (lieu et date de livraison finale).

Les HO, qui encapsulent un HP ou qui agrègent un ensemble de HO, et les HR vont donc collaborer pour élaborer au sein d'une holarchie $a d$ hoc une solution commune, tout en ayant des objectifs qui leur soient propres. Un $\mathrm{HR}_{\mathrm{j}}$ cherchera forcément à remplir sa capacité de transport avant sa date de départ $t_{d e p, l, j}$ sur un segment $\mathrm{S}_{1}$ donné, tandis qu'un $\mathrm{HO}_{\mathrm{k}}$ aura à cœur de faire progresser le $\mathrm{HP}_{\mathrm{x}}$ dont il a la charge sur un chemin qui le rapproche de sa destination et qui lui permettra d'assurer la livraison dans les temps. Chaque holon a donc des objectifs qui lui sont propres (Cf. Figure 6).

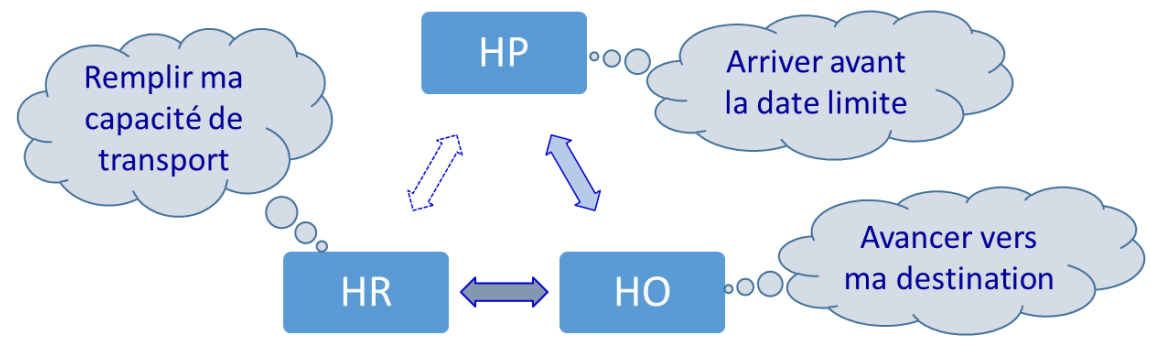

Figure 6. Interactions dans un contexte multi objectif

Si nous nommons $C_{a, b}$ le chemin pour aller du Hub de départ $H_{a}$ au Hub de destination $H_{b}$, ce chemin est une suite donnée de segments $S_{l}$ tels que $C_{a, b}=\mathrm{U}_{l=1}^{n} S_{l}$ avec :

- $S_{l}$ le segment entre le Hub de départ $H_{l a}$ et le Hub d'arrivée $H_{l, b}$, soit $S_{l}=\left[H_{l, a}, H_{l, b}\right]$;

- la condition de départ : $H_{1, a}=H_{a}$;

- la condition de continuité du transport dans un Hub intermédiaire : $H_{l, b}=H_{l+l, a}$;

- la condition d'arrivée à destination : $H_{n, b}=H_{b}$.

Chaque $\mathrm{HR}_{\mathrm{j}}$ et chaque $\mathrm{HO}_{\mathrm{k}}$ procède par appel d'offres, segment par segment, auxquels répondent les holons intéressés. Plusieurs types de technologies de transport peuvent se trouver en concurrence sur un même segment, chacune ayant des forces, des inconvénients et des disponibilités. Les réponses, concurrentielles, sont analysées via une aide multicritère à la décision, ce qui permet de mettre en avant les conditions les plus avantageuses en sens plus général d'un compromis.

Ainsi, un $\mathrm{HR}_{\mathrm{j}}$ lance une offre de service de transport pour le segment $S_{l}$ avec comme date de départ $t_{\text {dep, }, l, j}$. De son coté, chaque $\mathrm{HO}_{\mathrm{k}}$ reçoit différentes offres de service de transport qui peuvent lui convenir. Ces offres diffèrent en termes de dates de départ, de rapprochement de la destination finale, de marge restante vis-à-vis de la date de livraison, de prix, de durabilité du mode de transport utilisé, ou pourquoi pas, de fiabilité et de réputation de la compagnie organisant le transport. $\mathrm{Le} \mathrm{HO}_{\mathrm{k}}$ procède alors à une analyse multicritère qui lui permet de classer ces offres de transport, la première classée 
étant celle qui présente le meilleur compromis entre ses propres exigences. Il tente alors une réponse auprès du $\mathrm{HR}_{\mathrm{j}}$ concerné.

Ce dernier reçoit un ensemble de réponses. Si cet ensemble de réponses représente un volume de PIcontainers qui dépasse sa propre capacité, il procède à une analyse multicritère pour classer les demandes de transport prioritaires. Le $\mathrm{HR}_{\mathrm{j}}$ choisit alors les $\mathrm{p}$ premières réponses classées qui lui permettent de remplir sa propre capacité, informe les $\mathrm{HO}_{\mathrm{k}}$ correspondant qu'ils ont été retenus, et informe les autres $\mathrm{HO}_{\mathrm{k}}$ demandeurs du refus de leur demande.

Ces derniers relancent alors le processus de recherche multicritère d'un $H R$, en excluant $H R_{j}$ de l'ensemble des possibilités.

Au-delà de ces règles permettant d'assurer le fonctionnement auto-organisé nominal d'un système logistique global tel que l'Internet Physique, nous pouvons envisager d'autres règles permettant d'en accroitre la flexibilité et la réactivité, à l'instar de solutions déjà testées dans [OUN 12], [PUJ 16]. Ainsi, il est possible à un $\mathrm{HO}$ d'anticiper sur ses déplacements sur les segments à venir, en répondant de manière prévisionnelle à des HR et en se pré-engageant sur certaines offres. Ainsi, s'il reçoit une meilleure offre d'un nouveau HR, il peut libérer celui qu'il avait précédemment choisi. De même, si un HR a un besoin urgent de capacité, pour prioriser un transport urgent, il peut chercher parmi les HO qu'il a accepté celui qui accepterait de rechercher un nouveau moyen de transport...

Nous voyons maintenant clairement le rôle fondamental joué par le mécanisme d'aide multicritère à la décision : chaque holon peut le solliciter face à un choix difficile, et les informations à considérer lors de la mise en œuvre de l'algorithme sont des données et/ou des variables relatives aux autres holons de la holarchie dans laquelle se pose la question de ce choix.

Par ailleurs, si l'obtention d'un résultat de classement ANP est relativement rapide (nombre d'opérations arithmétiques: $\mathrm{k} \cdot \mathrm{O}\left(\mathrm{n}^{3}\right)$ ), il n'en demeure pas moins que cela demande une certaine puissance de calcul, et ceci n'est pas forcément compatible avec les technologies permettant de mettre en œuvre actuellement les CPLS.

Aussi, les CPLS ayant d'excellentes dispositions à la communication, nous proposons de mettre en place un usage distant de cette aide multicritère à la décision.

Pour cela, l'algorithme de mise en œuvre d'ANP va être codé de manière à être totalement générique. Chaque sollicitation du service distant ANP va induire de construire automatiquement la structure mathématique de calcul, en fonction des dimensions du problème à résoudre et du nombre d'alternatives en concurrence (c'est-à-dire l'ensemble des holons entre lesquels il faut choisir et qui appartiennent à la holarchie où se pose la question de ce choix). Chaque sollicitation indiquera donc la structure décisionnelle utilisée, le jeu de paramétrages par paires choisi et l'holarchie concernée. Bien entendu, ces éléments de structures décisionnelles, de pondérations de choix et d'existences d'holarchies doivent avoir été définis initialement lors de la configuration du système de pilotage. Fort de ces informations, le service distant ANP va pouvoir s'exécuter de manière spécifique vis-à-vis du problème posé et retourner au holon demandeur un classement qui lui permettra de pouvoir prendre sa décision.

La méthode ANP s'appuie, d'une part, sur une structuration des composantes importantes du problème (critères, indicateurs, alternatives (solutions possibles)), et d'autre part, sur une série de comparaisons deux à deux. Il est donc nécessaire de régler au préalable le paramétrage des différentes pondérations de comparaison entre critères et entre indicateurs. La configuration de la méthode s'effectue en amont de la phase d'exploitation temps réel dans le but de valider la cohérence de ces pondérations. Ces pondérations sont élaborées par un décideur (expert ou groupe d'experts). C'est un travail délicat car il faut définir la bonne structure décisionnelle et les pondérations adéquates. [OUN 18] a montré qu'il existe un très large éventail de positionnement décisionnel quant à cette 
problématique. ANP utilise donc un jeu de paramètres, estimés par expertise avec une possible marge d'incertitude. Dans le but d'optimiser cette phase d'identification des pondérations, une métaheuristique (un algorithme génétique) a été exploitée pour tester des configurations de paramètres proches de celle proposée par l'expert [OUN 13]. Ceci permet d'identifier la meilleure configuration possible et d'améliorer ainsi le résultat de l'aide à la décision.

Cette phase de configuration permet donc d'identifier les éléments structurant la décision. Il est ensuite possible de lancer la phase d'exploitation de la méthode d'aide multicritère à la décision qui permet d'obtenir un classement des alternatives et de mettre en évidence la tâche à réaliser. En phase d'exploitation, l'appel à l'aide multicritère à la décision s'effectue un peu avant le début de chaque tâche. Ce délai peut s'assimiler à un délai de préparation de la tâche. Ce délai peut être relativement court. Il est à ajuster en fonction de l'état d'occupation de l'ensemble des ressources concernées de telle manière à pouvoir obtenir la disponibilité de la ressource nécessaire à l'exécution de la tâche. Prenons l'exemple d'un bateau qui arrive à un port. Cela impliquera le déchargement de conteneurs. Chaque conteneur a besoin de lancer un appel au service d'aide multicritère à la décision pour identifier le camion qui le transportera. Cet appel sera effectué quelque temps avant l'arrivée au port. Ce délai sera fonction du taux d'occupation des camions. Cette donnée est à ajuster comme paramètre de fonctionnent du système de pilotage, que l'on soit dans le cas d'une simulation ou d'une exploitation réelle.

\section{Opérationnalisation}

Les mécanismes complexes que nous venons de décrire rapidement constituent les fondements de l'intelligence associée à chaque holon. Si ces mécanismes sont spécifiques à chaque type de holon ( $\mathrm{PH}$, $\mathrm{RH}$ et $\mathrm{OH}$ ) et paramétrés à façon sur chaque holon, il n'en demeure pas moins que les principes de fonctionnement sont commun à tous : le principe est que le système logistique global est un CPLS luimême composé de manière récursive de CPLS, jusqu'à atteindre les CPLS élémentaires qui correspondent aux holons les plus simples : produit à transporter, ressources de base de transport (camion, train, porte-containers...) et PI-containers isolés.

Nous allons maintenant décrire comment passer du paradigme holonique à l'opérationnalisation d'un CPLS et présenter un des cas d'application qui nous sert de support pour la mise au point d'un démonstrateur.

\subsection{Opérationnalisation d'un CPLS : implémentation vs simulation}

Pour passer d'un holon conceptuel à un CPLS opérationnel, il faut associer à sa partie physique, le M_holon, un calculateur embarqué, doté de capacité de communication, qui assurera la gestion des traitements numériques supportés par le I_holon. Différentes plateformes d'électronique embarquée sont susceptibles de répondre à cette exigence pour implémenter réellement des démonstrateurs de CPLS : cartes de type motes WSN (Wireless Sensor Network), cartes de type Arduino, Raspberry PI, ou ESP8266...

Le I_holon peut ainsi assurer les différentes fonctions nécessaires au fonctionnement d'un CPLS : une machine à état (assurant le cœur du fonctionnement du CPLS, en spécialisant les traitements en fonction de l'étape en cours dans les mécanismes décisionnels précédemment décrits), une fonction de communication (pour interagir avec les autres I_holons, qu'ils soient proches ou distants, en utilisant le relai d'un serveur par exemple), une fonction d'appel à des services distants (par exemple, un Web service capable de faire une analyse multicritère ou un autre capable de trouver un itinéraire...) ou des fonctions locales de perception (mesure de températures pour un PI-container réfrigéré, mesure de localisation via un GPS embarqué...)... Ces différentes fonctions nécessitent un traitement temps réel en multi thread. 
Les codes étant relativement génériques, la récursivité des holons peut être gérée dans les CPLS élémentaires. Par exemple, le I_holon du $\mathrm{OH}_{\mathrm{m}}$ agrégeant l'ensemble de holons $\left\{\mathrm{OH}_{\mathrm{k}}\right\}_{\mathrm{m}}$ peut être hébergé par le calculateur embarqué de l'un des $\mathrm{OH}_{\mathrm{k}}$, en fonctionnant avec les mêmes algorithmes, mais avec le paramétrage adapté. Lors de la décomposition du PI-conteneur en PI-conteneurs plus petits, le calculateur hôte retrouvera son I_holon d'origine.

Ce mécanisme est possible à implémenter dans la mesure où les agrégations de holons se font type par type, et que donc le fonctionnement reste globalement le même, seul le paramétrage de ce fonctionnement étant différent. Ainsi, le cœur du code interne, le fonctionnement du graphe d'état correspondant à un type donné de holon (HP, HR ou HO) reste strictement identique quel que soit le niveau d'agrégation/récursivité considéré.

Par exemple, la figure 7 montre 2 exemples de Statechart montrant une partie du fonctionnement d'un HR : la gestion d'un déplacement et la gestion d'un remplissage de capacité.

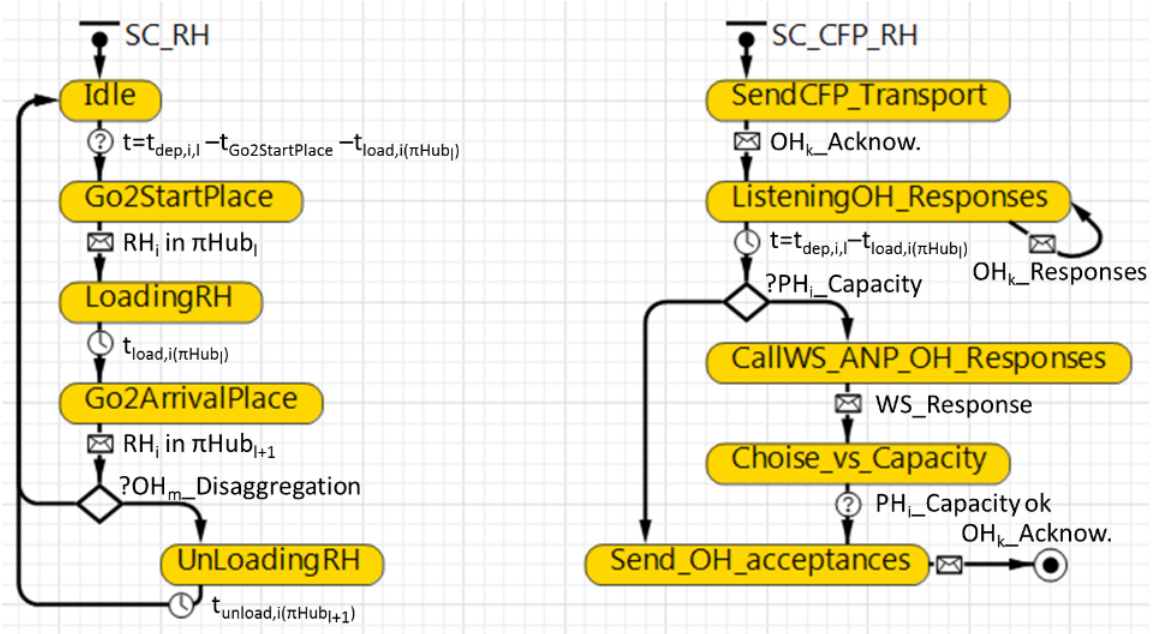

Figure 7. Exemples de Statecharts d'un HR

Au-delà de l'opérationnalisation réelle des CPLS, et donc de leur implantation sur des systèmes embarqués, nous avons prévu, pour des considérations pratiques, d'utiliser la simulation industrielle comme outil de développement et de mise au point de nos algorithmes. Pour cela, nous avons fait le choix de développer une plateforme de démonstration hybride de CPLS, c'est-à-dire capable de fonctionner en simulation et/ou en interaction de CPLS réels.

\subsection{Présentation de l'architecture du démonstrateur : simulation et web service ANP}

La plateforme de démonstration hybride a comme objectif de permettre un fonctionnement conjoint ou indépendant de solutions CPLS réelles ou simulées. Nous avons choisi un logiciel de simulation industrielle (Anylogic) supportant différents formalismes de modélisation, et entre autres l'approche multi-agents et les Statecharts, ce qui permet d'implémenter dans le modèle de simulation de chaque agent correspondant à un type de holon exactement le même graphe d'état que celui codé dans le calculateur embarqué d'un CPLS de ce type. De même, le service distant ANP d'aide multicritère à la décision peut être appelé indifféremment par un agent du logiciel de simulation et/ou par le calculateur embarqué d'un CPLS.

Pour cela, nous avons développé une architecture client-serveur adaptée à nos besoins, qui permet de connecter tous les acteurs, qu'ils soient réels ou simulés, autour d'un même protocole de communication incluant l'échange d'informations et l'appel à des services distants (Cf. Figure 8). Cette plateforme, conçue pour être la plus générique possible, doit être capable de traiter toutes sortes de CPLS abordant l'Internet Physique selon notre approche auto-organisée et isoarchique. Elle est organisée autour d'un serveur de sockets, qui d'une part gère les communications engendrées par les 
interactions entre les CPLS, ceux réellement implémentés et/ou ceux en cours de simulation, et d'autre part, relaie les appels au service distant ANP et les réponses de ce dernier.

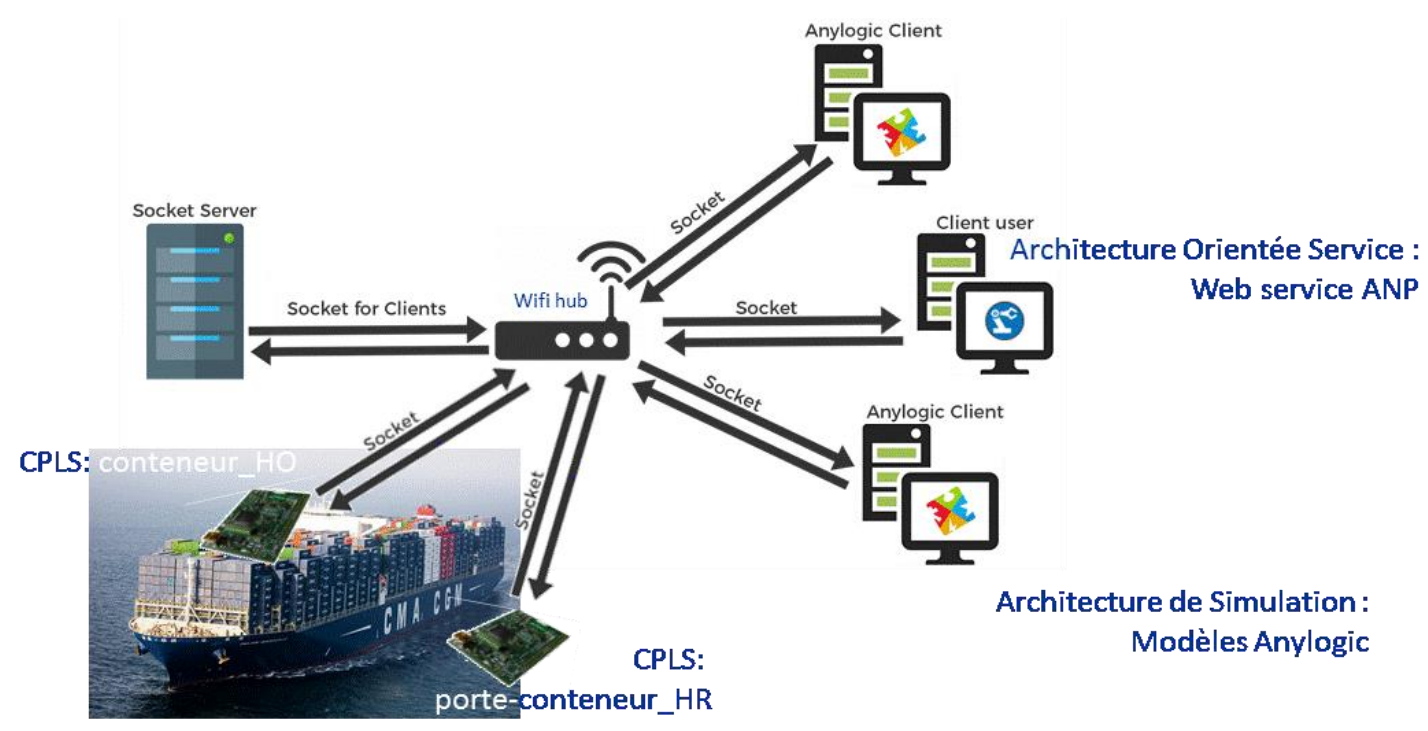

Figure 8. Architecture client-serveur de la plateforme de démonstration hybride

Ce service distant ANP est en fait un Web service implanté sur un serveur d'application Tomcat 8.

Pour illustrer les principes énoncés ici, nous avons choisi le cas du transit de conteneurs entre la Chine et la France.

\subsection{Cas du transport intercontinental de conteneurs : Chine - France}

Le trafic de conteneurs entre ces 2 pays est important. Par hypothèse, nous l'avons artificiellement isolé du reste du trafic intercontinental, afin de simplifier un peu les problématiques à gérer. Il existe une concurrence importante sur ce marché, et c'est ce qui présente un intérêt majeur pour tester des solutions d'auto-organisation en logistique. La carte (Cf. Figure 9) permet de visualiser les différentes alternatives en concurrence : une ligne par rail, en passant par la Russie et l'Europe du nord, entre des gares chinoises (GC) et une gare française (GF) située à Lyon, et plusieurs lignes maritimes opérées par différentes compagnies entre des ports chinois (PC) et 2 ports français $(\mathrm{PF})$.

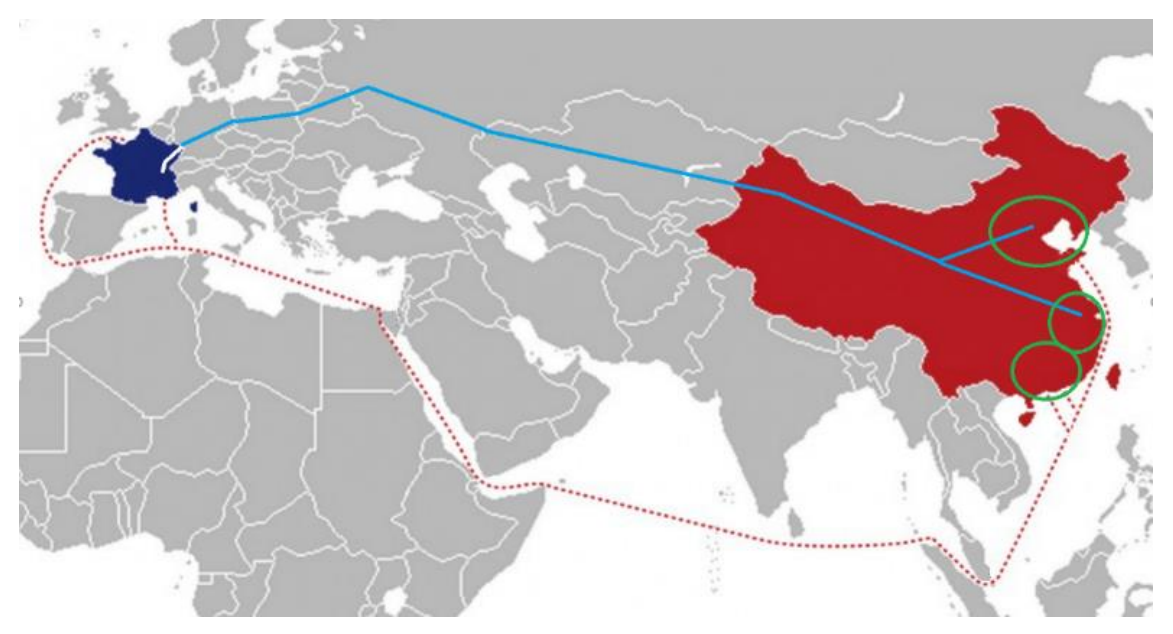

Figure 9. Visualisation géographique des routes maritimes et ferroviaires

Globalement, nous pouvons dire que le train est plus rapide que le bateau et que les conditions économiques et écologiques sont plus ou moins convergentes [SEV 11]. En effet, comme le transport maritime répond à une exigence de massification des flux, il est considéré comme un mode de 
transport 'écologique' : il consomme le moins d'énergie et émet le moins de gaz à effet de serre par tonne-kilomètre. Cependant, cette idée reçue dépend du type de navire utilisé. Par ailleurs, au vu des réglementations mises en place sur le plan de la maitrise de la pollution de l'air, le transport routier rivalise avec le transport maritime. Cela conduit à considérer le transport maritime comme étant globalement le mode de transport le moins polluant en émissions de gaz à effet de serre. Cela n'est pas le cas pour les polluants de l'air (oxydes de soufre, oxydes d'azote, particules en suspension) [DOU 15].

Cette concurrence entre différents CPLS de transit Chine-France sera auto-organisée via une structure ANP telle que celle présentée en figure 10.

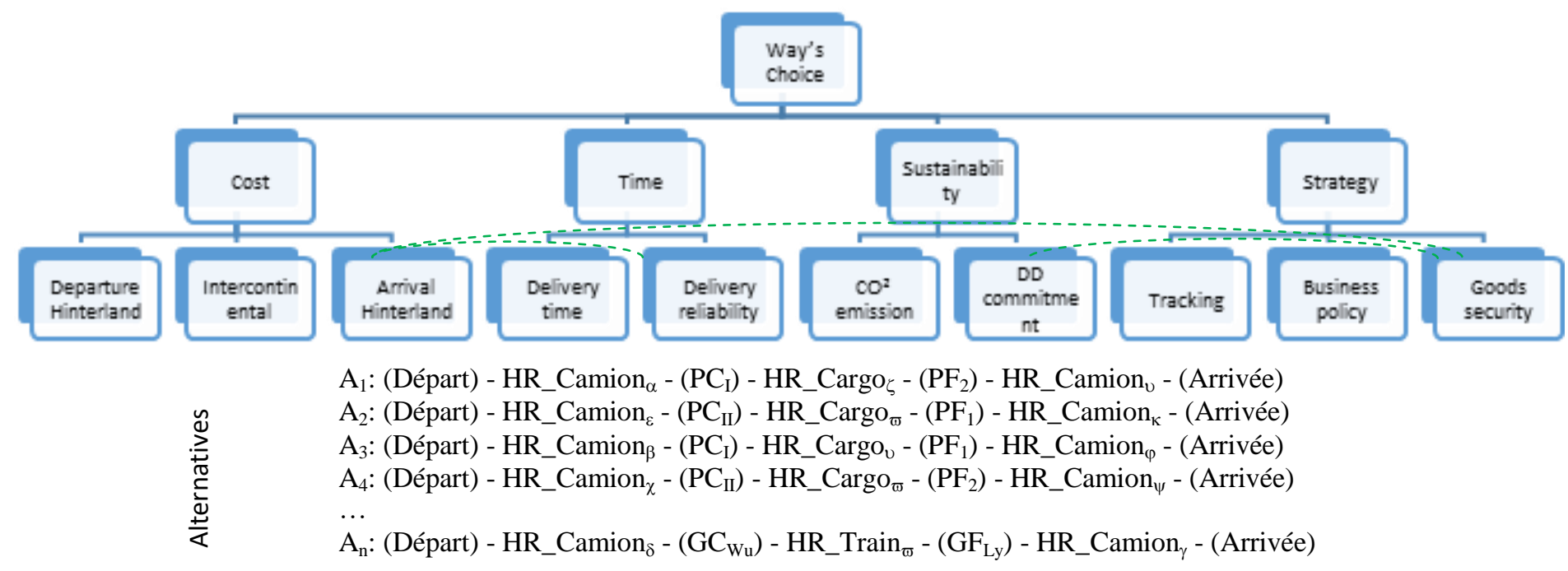

Figure 10. Structure de décision ANP entre différentes alternatives de transport

Cette structure décisionnelle couvre des aspects divers, complémentaires et parfois contradictoires tels que les coûts (au départ, en intercontinental et à l'arrivée), le respect des délais (date et fiabilité), la stratégie (commerciale, éthique) et le développement durable (émissions...).

En effet, chaque Holon Ordre (HO) choisira le 'bon' chemin pour transporter le PI-container correspondant, selon une analyse multicritères basée sur les critères suivants :

- le premier critère 'Coût' permet d'assurer une livraison au meilleur prix et prend en compte les différents coûts liés au transport de marchandises. Ce critère quantitatif est composé de trois indicateurs : l'hinterland de départ ' $\mathrm{I}_{11}$ ', l'intercontinental ' $\mathrm{I}_{12}$ ' et l'hinterland d'arrivée ' $\mathrm{I}_{13}$ ' ;

- l'objectif du deuxième critère 'Délai' est de faire en sorte que le PI-container soit livré le plus rapidement possible. Ce critère quantitatif peut être décomposé en deux indicateurs : délai de livraison ' $\mathrm{I}_{21}$ ' et fiabilité de livraison ' $\mathrm{I}_{22}$ ';

- le troisième critère 'Durabilité' garantit que le développement durable est l'un des objectifs du HR. Ce critère est composé de deux indicateurs : les émissions de $\mathrm{CO} 2$ ' $\mathrm{I}_{31}$ ' et l'engagement en développement durable ' $\mathrm{I}_{32}$ ';

- le dernier critère concerne les critères qualitatifs nommés 'Stratégie' liés, par exemple, à une relation privilégiée ou liés au respect de certaines conditions. Ce critère est composé de trois indicateurs : Traçabilité ' $\mathrm{I}_{41}$ ', Politique d'entreprise ' $\mathrm{I}_{42}$ ' et sécurité des biens ' $\mathrm{I}_{43}$ '.

L'organisation du transport a été décomposée en quatre 4 phases (Cf. Figure 11) :

- choix du chemin global de transport : choix du mode de transport (bateau ou train) et des lieux d'étapes principales associés (quels sont les hubs de groupage en Chine et de dégroupage en France) ; 
- choix du transport sur l'hinterland amont (départ): choix du transporteur qui amènera les conteneurs au port / à la gare précédemment défini(e) ;

- choix du transport intercontinental: choix du transporteur sur le chemin intercontinental (compagnie maritime ou ferroviaire) ;

- choix du transport sur l'hinterland aval (arrivée): choix du transporteur qui amènera les conteneurs du port / de la gare au client.

Dans le but d'enrichir la structure de décision, il sera possible de se baser sur les résultats d'une recherche bibliographique concernant les critères de choix de prestataires de services logistiques (PSL) [OUN 18]. Une revue systématique a été réalisée en se basant sur un travail de collecte, d'analyse et de synthèse des connaissances existantes afin de lister les critères les plus mobilisés par les acteurs.

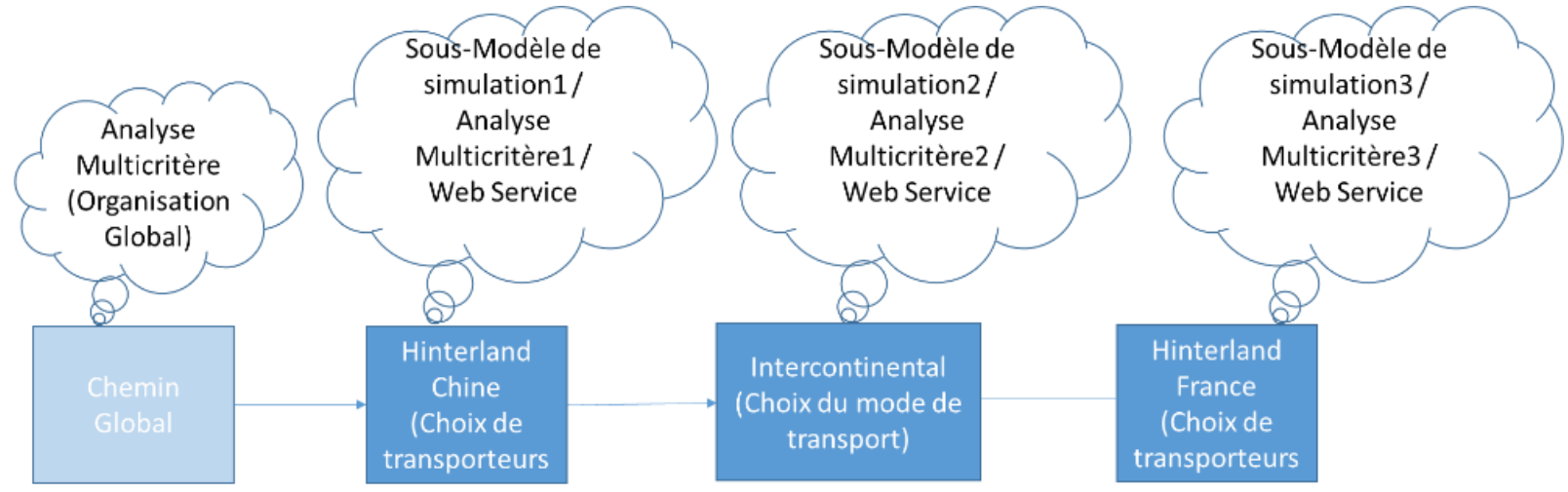

Figure 11. Organisation du transport

Pour conclure sur cet aspect, nous présentons une copie d'écran du modèle de simulation (Cf. Figure 12). Ce modle gère les 4 phases précédentes et intègre la totalité des parties prenantes concernées par ces échanges commerciaux. Ainsi, on y voit l'hinterland de départ, en Chine, où différentes implantations industrielles alimentent en conteneurs le flux Chine-France, soit via un des 3 ports chinois choisis ou la gare de fret du Wuhan, en utilisant différents prestataires logistiques eux-mêmes en concurrence sur ce marché du transport chinois. La meme mise en concurrence est aussi de mise sur l'hinterland de destination, en France.

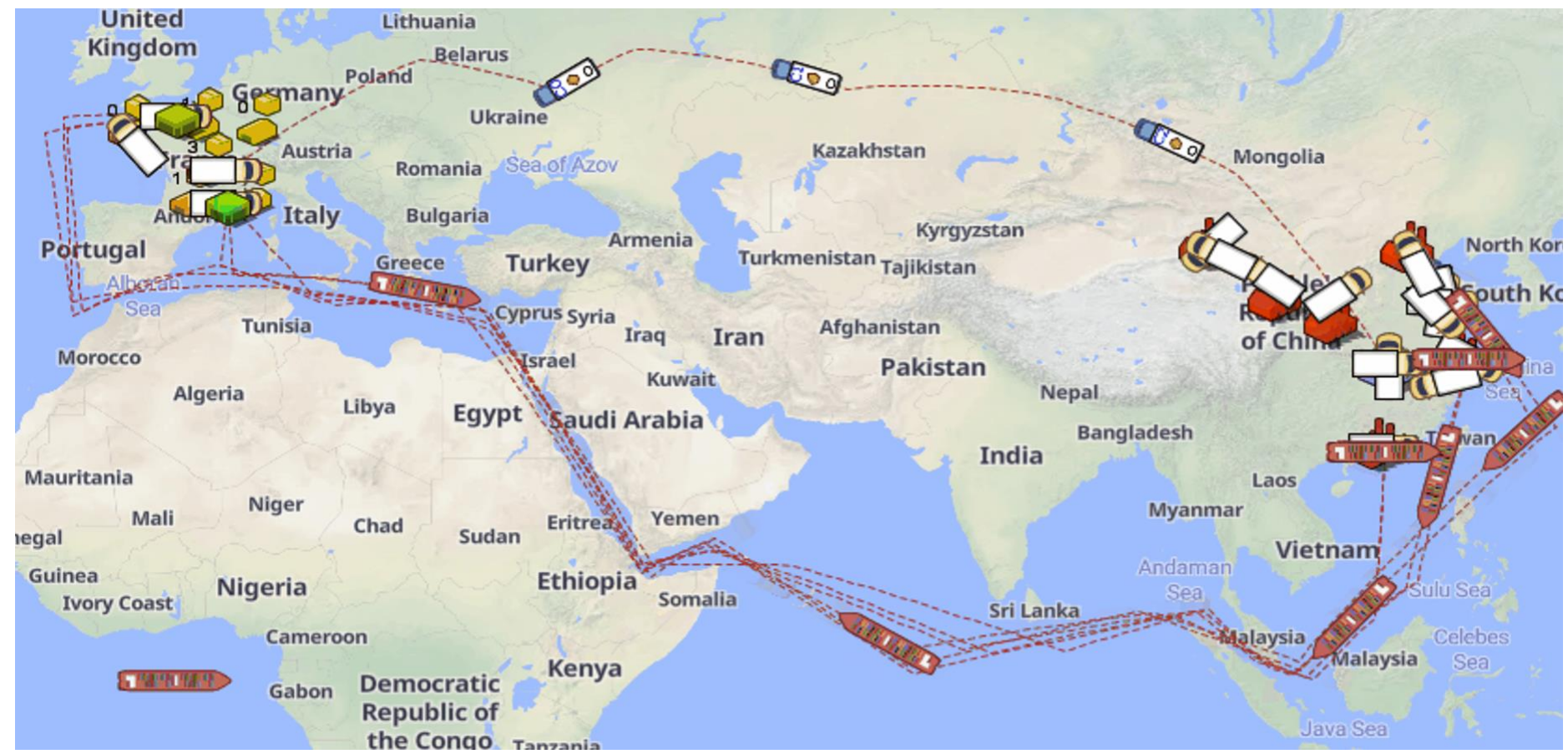

Figure 12. Copie d'écran de la simulation 


\section{Conclusion}

Face à la contingence des événements et à la dimension planétaire de l'apparition d'événements perturbateurs aléatoires, les solutions fondées sur l'organisation prévisionnelle posent plus de problèmes que ce qu'elles en résolvent.

Le caractère opportuniste d'une solution décisionnelle locale, temps réelle, multicritère permet un pragmatisme qui, même s'il n'est pas à priori optimal, génère de très bonnes solutions et élimine par construction toute situation de blocage.

Une proposition de pilotage d'un système logistique global basée sur l'Internet Physique, les CPLS et le paradigme holonique, est décrite. Chaque entité holonique est un CPLS appartenant à un CPLS plus global. Un CPLS est donc un holon implémenté. En effet, chaque entité physique est associée à un ordinateur embarqué, qui supporte son intelligence associée. Chaque CPLS appartient à un réseau dans lequel les décisions collectives émergent sur la base d'une aide multicritère à la décision. Dans ce contexte, il faut toujours qu'il y ait une certaine adéquation entre la charge demandée au réseau logistique auto-organisée et sa capacité globale. Cette capacité résulte de l'agrégation des capacités moyennes des PSL qui adhèrent au fonctionnement proposé. Si la charge requise, résultant des demandes cumulées des clients utilisateurs, augmente ou décroit, il faut alors prévoir un ajustement de cette capacité globale. Cette recherche de l'adéquation peut être faite, par exemple, par l'ajout de nouvelles capacités de ressources de transport par les PSL existants ou bien par l'intégration de nouveaux PSL partenaires dans le réseau logistique auto-organisé. Dans tous les cas, les PSL les plus performants seront ceux qui rempliront le plus rapidement leur planning de charge. Ils seront donc tentés d'augmenter leur capacité. Les PSL les moins performants se retrouvent, quant à eux, plus ou moins en situation de variable d'ajustement. A charge pour eux d'augmenter leurs performances (fiabilité, délai, coût, qualité, ...), pour augmenter leur niveau de qualité de service et pouvoir proposer une offre plus en adéquation avec les besoins des clients. Cela leur permettrait de sortir de cette situation de PSL 'd'ajustement'.

Il s'agira pour la suite de mettre en place des expérimentations réalistes pour établir factuellement les avantages des réseaux logistiques dynamiques liés à la robustesse de leur comportement.

\section{Bibliographie}

[BAL 01] BALASUbramanian S., BREnNAN R., NORRIE D., «An architecture for metamorphic control of Holonic Manufacturing Systems », Computers in Industry, n 46, p. 13-31, 2001.

[BAL 12] Ballot E., Gobet O., Montreuil B., «Physical internet enabled open hub network design for distributed networked operations, Service orientation in holonic and multi-agent manufacturing control. Springer, p. 279-292, 2012.

[BAL 13] Ballot E., Montreuil B., Thivierge C., Functional design of physical internet facilities: a road-rail hub, CIRRELT, 2013.

[BAL 14] BALlot E., Montreuil B., L'Internet Physique, La documentation française, 2014.

[CAR 17] CARDin O., Ounnar F., Thomas A., Trentesaux D., «Future Industrial Systems: Best Practices of the Intelligent Manufacturing and Services Systems (IMS2) French Research Group », IEEE Transactions on Industrial Informatics, $\mathrm{n}^{\circ} 13$ (2), p. 704-713, 2017.

[DEE 03] DEEN S.M., Agent-Based Manufacturing - Advances in the Holonic Approach, Springer-Verlag, ISBN 3-54044069-0, 2003.

[DOU 02] Doumpos M., ZoPOUNIDIS C., Multicriteria decision aid classification methods, Kluwer AP, 2002.

[DOU 15] DOUDNIKOFF M., Réduire les émissions du transport maritime : les politiques publiques et leurs impacts sur les stratégies des compagnies maritimes de lignes régulières, Thèse de doctorat en Economies et finances. Université Paris-Est, 2015. 
[ELK 16] El KadiRi S., Grabot B., ThOBEn K.D., HRIBERniK K., EMMANOUILIDIS C., Von CiEMINSKi G., KiRITSIS D., « Current trends on ICT technologies for enterprise information systems », Computers in Industry, ${ }^{\circ}$ 79, p. 14-33, 2016.

[FUL 11] FUlCONIS F., PACHE G., RoveILlo G., La prestation logistique. Origines, enjeux et perspectives, EMS, 2011.

[HER 16] HeRmann M., PenteK T., OtTo B., « Design principles for industry 4.0 scenarios: A Literature Review », $49^{\text {th }}$ Hawaii International Conference on System Sciences (HICSS). IEEE, 2016.

[HOU 15] Hoummady M., Lemercier P., BoISARD A.S., Evolutions de la logistique à l'horizon 2025 : enjeux et impacts du Big data, de l'Intelligence Artificielle et de la Robotisation, Rapport de mission. Conférence nationale sur la logistique 2025. Paris, 2015.

[ISH 13] ISHIZAKA A., NeMERY P., Multi-Criteria Decision Analysis: Methods \& Software, Wiley, 2013.

[KAG 13] Kagermann H., WAHLSTER W., HelBig J., Recommendations for implementing the strategic initiative Industry 4.0, Final report of the Industry 4.0 Working Group, 2013.

[KOE 67] KOESTLER A., The ghost in the machine, Editions Hutchinson, London, 1967.

[KOH 15] KOHLER D., WeISZ J.D., Industrie 4.0 : quelles stratégies numériques ? La numérisation de l'industrie dans les entreprises du Mittelstand allemand, Publication BPI-France Industrie 4.0, Kohler Consulting \& Coaching, 2015.

[LAI 11] LaI M.Y., Jin C.F., NIE K., ZHAO J.H., « Cyber Physical Logistics System: The Implementation and Challenges of Next-generation Logistics System », Systems Engineering, n 4, 008, 2011.

[LEE 08] LEE E.A., « Cyber physical systems: Design challenges », 11th IEEE International Symposium on Object and Component-Oriented Real-Time Distributed Computing (ISORC), p. 363-369. IEEE, 2008.

[LEI 06] LEITÃo P., RESTIVO F., «ADACOR: a holonic architecture for agile and adaptive manufacturing control », Computers in Industry, $\mathrm{n}^{\circ}$ 57, p. 121-130, 2006.

[LEI 09] LEITÃO P., «Agent-based distributed manufacturing control: A state-of-the-art survey », Engineering Applications of Artificial Intelligence, $\mathrm{n}^{\circ}$ 22(7) , p. 979-991, 2009.

[LEI 16] Leitão P., Colombo A.W., KARnouskos S., «Industrial automation based on cyber-physical systems technologies: Prototype implementations and challenges », Computers in Industry, n 81, p. 11-25, 2016.

[LEM 90] Le Moigne J.L., La modélisation des systèmes complexes, Paris : Bordas, Dunod, 1990.

[MON 06] MONOSTORI L., VAnCZA J., KUMARA S.R.T,. «Agent-Based Systems for Manufacturing”. CIRP Annals Manufacturing Technology, ${ }^{\circ} 55$ (2), p. 697-720, 2006.

[MON 11] MONTREUIL B., «Towards a Physical Internet: Meeting the Global Logistics Sustainability Grand Challenge », Logistics Research, $\mathrm{n}^{\circ} 3$ (2-3), p. 71-87, 2011.

[MON 14] MonOstoRi L., « Cyber-physical production systems: Roots, expectations and R\&D challenges », Procedia CIRP, n 17, p. 9-13, 2014.

[MON 15] MONOSTORI L., « Cyber-physical production and logistics systems: Roots, expectations, R\&D challenges and results », 37 $7^{\text {th }}$ AIM Conference. Vienna, Austria, September 19, 2015.

[NOY 16] NOYER J.M., «L'Internet des Objets, l'Internet of "Everything": quelques remarques sur l'intensification du plissement numérique du monde », Open Science-Internet des objets, $\mathrm{n}^{\circ}$ 17(1), 2016.

[OLI 97] Oliver D.W., Kelliher T.P., Keegan J.G., Engineering Complex Systems, McGraw-Hill Companies, 1997.

[OUN 08] OUNNAR F., Pujo P., Isoarchic and Multi-criteria Control of Supply Chain Network, In: Artificial Intelligence Techniques for Networked Manufacturing Enterprises Management (Chap 6), Springer-Verlag, 2008.

[OUN 12] OUNNAR F., PUJO P., « Pull control for job shop: holonic manufacturing system approach using multicriteria decision-making », Journal of Intelligent Manufacturing, $\mathrm{n}^{\circ}$ 23, p. 141-153, 2012.

[OUN 13] OUNNAR F., PUJO P., DENGUIR A., Optimization via a genetic algorithm parametrizing the AHP method for multicriteria workshop scheduling, In: Metaheuristics for Production Scheduling (Part 2), ISTE-Wiley, p. 119-150, 2013.

[OUN 15] OunNaR F., Pujo P., Hachicha M., Dubromelle Y., « Study of an intelligent and multicriteria scheduling service, using academic benchmarks », Int. J. of Computer Integrated Manufacturing, n 29, p. 1166-1176, 2015.

[OUN 16] OUNNAR F., PUjo P., « Holonic Logistics System: a novel point of view for Physical Internet », Proceedings of International Conference on Information Systems, Logistics and Supply Chain (ILS 2016), June 1-4, Bordeaux, 2016. 
[OUN 18] OUNNAR F., PUJO P., «Une formulation multicritère pour le choix de PSL dans un réseau partenarial autoorganisé », Logistique \& Management, n² 26/ 4, p. 289-301, 2018.

[PUJ 01] PUjO P., OUNNAR F., «Proposition d'un pilotage décentralisé, auto-organisé et rapproché pour système automatisé flexible : Application à un Hub de transbordement robotisé fer-fer », Journal Européen des Systèmes Automatisés, $\mathrm{n}^{\circ}$ 35, p. 905-932, 2001.

[PUJ 07] PUjo P., OUNNAR F., «Vers une approche holonique des Systèmes Mécatroniques Complexes : proposition d'un système de pilotage auto organisé et isoarchique », Journal Européen des Systèmes Automatisés, n 41, p. 673 706, 2007.

[PUJ 09] PUJO P., BROISSIN N., OUNNAR F., «PROSIS: An isoarchic structure for HMS control », Engineering Applications of Artificial Intelligence, $\mathrm{n}^{\circ} 22$ (7), p. 1034-1045, 2009.

[PUJ 12] PUjo P., Pilotage isoarchique des systems de production, EME, 2012.

[PUJ 16] Pujo P., Ounnar F., Power D., Khader S., «Wireless Holon Network for job shop isoarchic control », Computers in Industry, $\mathrm{n}^{\circ}$ 83, p. 12-27, 2016.

[SAA 96] SAATY T.L., Decision Making with Dependence and Feedback: The Analytic Network Process, RWS Publications, Pittsburgh, PA, 1996.

[SAL 18] SALEH I., «Internet des Objets (IdO): Concepts, Enjeux, Défis et Perspectives », Revue Internet des objets, $\mathrm{n}^{\circ} 2$ (10.21494), 2018.

[SAL 15] Sallez Y., Berger T., Bonte T., TrentesauX D., «Proposition of a hybrid control architecture for the routing in a Physical Internet cross-docking hub », IFAC-PapersOnLine, n 48 (3), p. 1978-1983, 2015.

[SEV 11] SEVIN J.C., La desserte maritime et terrestre de l'Europe en trafics conteneurisés à l'horizon 2030, Thèse de doctorat, CNAM, Paris, 2011.

[TAO 17] TAO F., Zhang M., ChEng J., « Digital twin workshop: a new paradigm for future workshop », Computer Integrated Manufacturing Systems, $\mathrm{n}^{\circ} 23$ (1), p. 1-9, 2017.

[THO 14] Thoben K.D., Veigt M., LapPe D., Franke M., KÜCK M., Kolberg D., FAHL I., Zimmerling R., Schlick J., STEPHAN P., GUTH P., « Towards Networking Logistics Resources to Enable a Demand-Driven Material Supply for Lean Production Systems - Basic Concept and Potential of a Cyber-Physical Logistics System », Proceedings of the $7^{\text {th }}$ Int. Scientific Symposium on Logistics, June 4-5, Bremen, p. 45-71, 2014.

[TRE 16] TRentesauX D., BorangiU Th., Thomas A., «Emerging ICT concepts for smart, safe and sustainable industrial systems », Computers in Industry, n 81, p. 1-10, 2016.

[VAN 98] Van Brussel H., Wyns J., Valckenaers P., Bongaerts L., Peeters P., « Reference architecture for holonic manufacturing systems: PROSA », Computers in Industry, n 37, p. 255-274, 1998.

[VER 01] VERBRAECK A., VERSTEEGT C., «Logistic control for fully automated large scale freight transport systems: event based control for the underground logistic system schiphol », Proceedings of Intelligent Transportation Systems IEEE conference, p. 768-773, 2001. 